\title{
Non-tropical pyomyositis
}

\author{
I MUSCAT, P P ANTHONY,* J G CRUICKSHANK \\ From the Departments of Microbiology and ${ }^{*}$ Histopathology, Royal Devon and Exeter Hospital, Exeter and \\ Public Health Laboratory, Exeter
}

SUmmary Pyomyositis occurred in a man who had not been to the tropics. The condition is com- $\vec{\circ}$ mon in the tropics but most unusual in temperate climates and is nearly always caused by Staphylococcus aureus. Pyomyositis must be borne in mind in obscure cases of sepsis, as early recognition $\vec{\omega}$ and treatment are essential to prevent a fatal outcome.

Voluntary muscle is remarkably resistant to blood borne bacterial infection. None the less, primary intramuscular abscesses are well recognised in the tropics, the condition being known as pyomyositis tropicans. ${ }^{1-3}$ Recently, it has been reported in temperate climates where its rarity often results in a delay in diagnosis. ${ }^{4-7}$ Progression to a toxaemic stage, though unusual, may be rapid and fatal. We report this case to draw attention to a condition that is eminently treatable if recognised in the early stages.

\section{Case report}

A 66 year old caucasian man was admitted to hospital with a four day history of right shoulder pain progressing to severe generalised myalgia. There was no history of trauma, travel in the tropics, or alcohol abuse. His medical history consisted of transient cerebral ischaemic attacks following a silent myocardial infarct three months previously and gout for nine years.

On examination he was pale, peripherally cyanosed, and tachypnoeic. His temperature was $36^{\circ} \mathrm{C}$, pulse 110 regular, blood pressure $100 / 60 \mathrm{~mm}$ $\mathrm{Hg}$. He had pitting oedema to his knees, and basal crepitations were heard in both lung fields. There was browny oedema around his right shoulder and the muscles of his trunk. The proximal parts of his limbs were tender with painful limitation of movement. His haemoglobin concentration was $15 \mathrm{~g} / \mathrm{dl}$, white cell count $8 \times 10^{9} / 1(80 \%$ polymorphs with left shift $)$, platelets $87 \times 10^{9} / 1$, and prothrombin time $16 \mathrm{sec}-$ onds (control 12 seconds). Blood urea concentration was $39 \mathrm{mmol} / 1(230 \mathrm{mg} / 100 \mathrm{ml})$ and he had a metabolic acidosis. He was also hypoxic. No abnormalities were seen on radiography of the right shoulder and no fluid was obtained on attempted aspiration of the

Accepted for publication 14 May 1986 joint. The chest radiograph showed diffuse patchy $\omega_{0}$ shadowing.

Septicaemia with pulmonary disease was diagnosed, and treatment with intravenous fluids, oxygen, $\vec{\exists}$ penicillin, flucloxacillin, fusidic acid, and gentamicin $\sigma$ was started. Despite cardiorespiratory support he 9 failed to respond and died 12 hours later. Staphy- $\vec{O}$ lococcus aureus (phage type 52) sensitive to flucloxacillin, fusidic acid, and gentamicin was iso- $\frac{0}{0}$ lated from all blood cultures taken on admission.

\section{Necropsy findings}

There were no external signs of injury. The right shoulder and left forearm were swollen. On exposure the muscles of the right upper arm, the left forearm, $\bar{D}$ and the left quadriceps were oedematous but showed

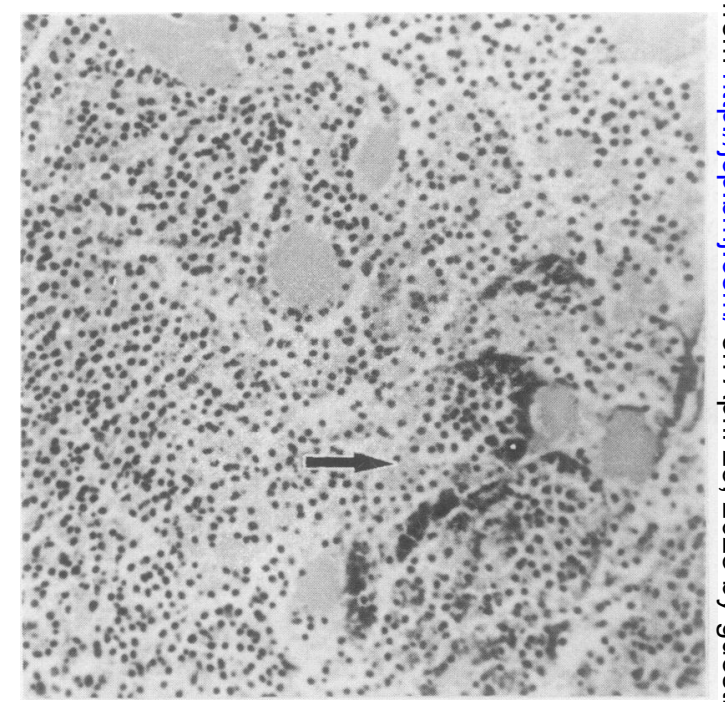

Fig 1 Small intramuscular abscess with darkly stained colonies of organisms. (Haematoxylin and eosin.) $\times 125$. 


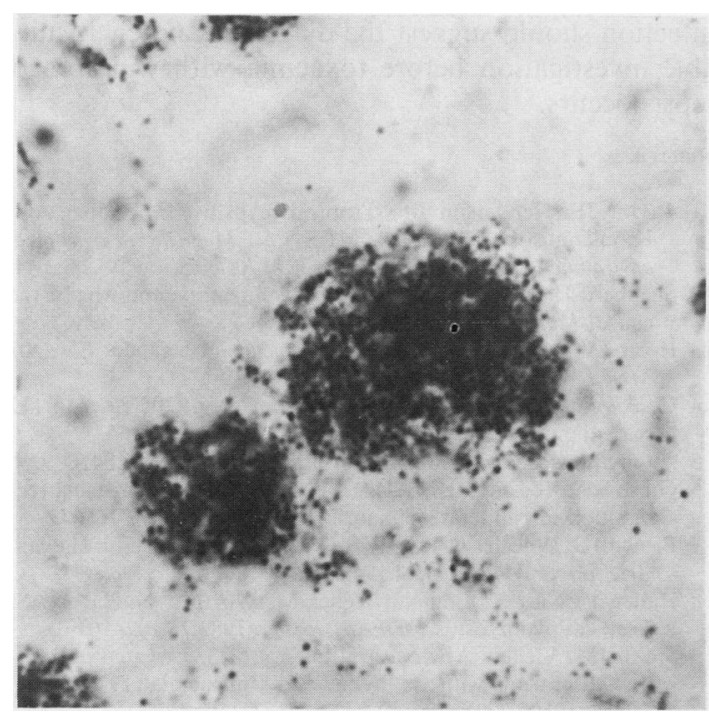

Fig 2 Colony of Gram positive cocci. $\times 800$.

no other change. The right shoulder joint was normal. The heart showed a three month old organising infarct and the rest of the myocardium was red. Multiple abscesses up to $1.5 \mathrm{~cm}$ in diameter were scattered throughout the lungs. The spleen was soft, the liver enlarged, and the kidneys oedematous and mottled. Sections of skeletal muscle and myocardium showed multiple foci of suppuration (fig 1), containing large numbers of Gram positive cocci (fig 2). These foci were intramuscular rather than between bundles or within fascial planes. Samples of muscle from unaffected sites were normal. The lung abscesses contained similar organisms and seemed to be septic infarcts (fig 3).

\section{Discussion}

Pyomyositis is a disease seen commonly in many tropical countries. It is characterised by the development of abscesses within skeletal muscle and may be associated with varying degrees of systemic disease. ${ }^{12}$ Staphylococcus aureus is isolated in more than $90 \%$ of cases. ${ }^{1-7}$ Most patients are healthy before the onset of infection and young males, often in the paediatric age group, predominate. Large muscle groups, like thigh, buttock, and shoulder are most commonly affected, and in $75 \%$ of cases only a single abscess is present. Pain in the affected muscle is the commonest presenting feature and is followed a few days to a week later by fever, local induration, oedema, and severe restriction of movement. Neutrophil leucocytosis is usually present. Blood cultures are only

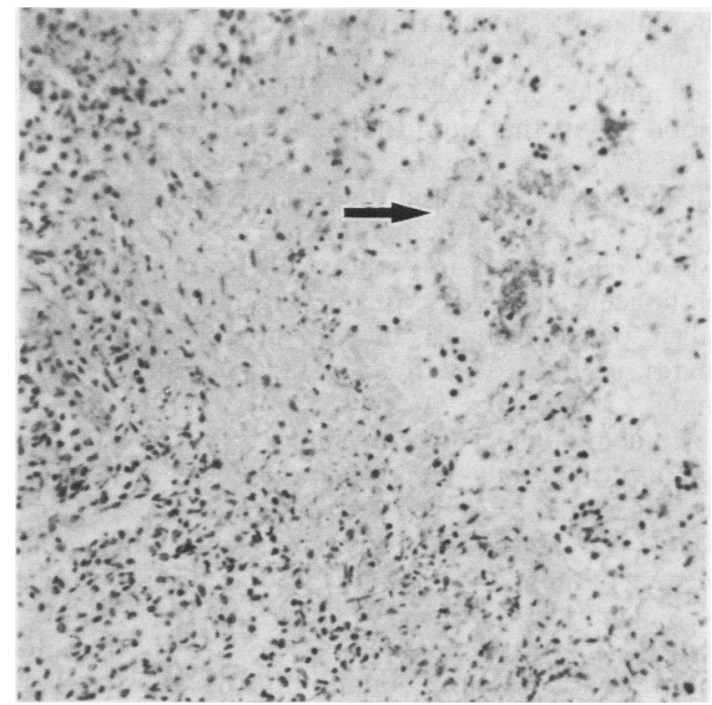

Fig 3 Edge of septic lung infarct with darkly stained colonies of organisms. (Haematoxylin and eosin.) $\times 125$.

positive in $5 \%$ of cases and muscle enzymes are rarely raised despite considerable muscle destruction. ${ }^{1-8}$ If not recognised and treated promptly frank septicaemia with the formation of metastatic abscesses will follow.

The disease differs from primary staphylococcal septicaemia, both in its clinical and pathological features. In three reviews encompassing 562 cases of Saureus septicaemia from various sources and portals of entry abscesses in skeletal muscle were found in only two, but details of distribution or risk factors were not documented. ${ }^{9-11}$

Pyomyositis is particularly common in east Africa and it has been reported to account for 3 to $4 \%$ of all surgical admissions to hospital in Uganda. ${ }^{3}$ It is rarely seen in temperate climates, ${ }^{125}$ but since the disease was first reported in the United States in $1971,{ }^{12}$ several cases have been recorded in American residents, and 31 of these were reviewed by Gibson et al. ${ }^{6}$ Of these, three cases were not considered to be consistent with the diagnosis, and three patients had recently returned from the tropics. Two had diabetes, ${ }^{613}$ two had a neutrophil polymorph count of $<250 \times 10^{9} / 1,{ }^{1415}$ and one was a heroin addict. ${ }^{16}$ Muscle disease was multiple in 16 patients and $S$ aureus was isolated from 23 of the 27 cases in which an organism was identified. Seven of 21 patients had positive blood cultures and two patients died.

Of the six cases of bacterial myositis reported in the British literature since 1930, four were of streptococcal origin and presented as rapidly developing cellulitis, ${ }^{17}$ and in another the abscess was inter- 
muscular rather than intramuscular. ${ }^{18}$ Williams and Thomas ${ }^{19}$ reported a case with acute disease of both calf muscles, in which swelling so compromised the blood supply that surgical intervention was needed to avert ischaemic damage. Saureus was isolated from the affected muscles and the patient recovered, albeit slowly, with antibiotic treatment. ${ }^{18}$

Saureus isolates in pyomyositis have no singular pathogenic properties. In one Ugandan series $70 \%$ fell in phage group II, but this group also predominates in deep seated abscesses in temperate climates. ${ }^{12}$

Epidemiological evidence suggests that host factors are important, but extensive studies have failed so far to explain the cause and distribution of the disease. ${ }^{2}$ Miyake $^{20}$ showed that in animals haematogenous seeding of muscle with $S$ aureus required prior trauma to that muscle. Trauma is reported in $20 \%$ of cases, but its importance is unknown. ${ }^{2-4621}$ Parasitic infestation is no more common in pyomyositis than would be expected from the local prevalence of infestation, and although nematode larvae were found in drained pus in one series, this has not been observed in other studies. ${ }^{422}$ The finding of muscle fibre degeneration and a mononuclear infiltrate in muscle at sites distant from abscesses suggest widespread muscle damage. ${ }^{2324}$ This and the rarity of recurrence of pyomyositis have suggested a viral ${ }^{23}$ or possibly a toxin induced predisposition. ${ }^{2}$ Neither theory has been confirmed, but it is possible that more than one specific factor may predispose muscle to bacterial infection. No abnormality was found in samples of unaffected muscle in our case.

In retrospect, our case had many of the features of the classically described disease but with rapid progression to septicaemia, metastatic abscesses, and death. None of the suggested predisposing factors was present, but the possibility exists that longstanding gout, or its treatment, may have resulted in some form of muscle damage, ${ }^{25}$ so providing the nidus on which transiently circulating organisms might have settled.

The differential diagnosis includes muscle trauma, deep vein thrombosis, osteomyelitis, and septic arthritis. If the axial musculature is affected pyomyositis may mimick intra-abdominal disease or pneumonia, and if multifocal, it needs to be distinguished from acute polymyositis and trichinosis. ${ }^{132627}$ Radioisotope scanning, computed tomography, and, most specifically, aspiration under ultrasonographic guidance have been claimed to be particularly helpful in diagnosis. ${ }^{368132628}$ Reports from the tropics show that with early drainage and appropriate antibiotics the mortality is less than $2 \% .{ }^{34}$ Further abscesses may present after treatment has begun but true recurrences are extremely rare and residual dysfunction or deformity uncommon. ${ }^{34}$

That the condition is rare in temperate countries is clear, but it does occur, and the presence of a localised or multifocal myalgia accompanied by features of infection should suggest the diagnosis and elicit suitable investigation before toxaemia with widespread sepsis occurs.

\section{References}

I Taylor JF, Henderson BF. Tropical myositis. In: Shaper AG. Kibukamusoke JW, Huttk MRS, eds. Medicine in a tropical environment. London: British Medical Association 1972:32-44.

2 Shepherd JJ. Tropical pyomyositis: is it an entity, and what is the cause? Lancet 1983;ii:1240-2.

3 Horn CV, Master S. Pyomyositis tropicans in Uganda. East Afr Med J 1968;45:463-71.

4 Chukwuma Chiedozi L. Pyomyositis: review of 205 cases in 112 patients. Am J Surg 1979;137:255-9.

5 Anonymous. Pyomyositis [Editorial]. Br Med J 1979;ii:458-9.

6 Gibson R, Rosenthal J, Lukert BP. Pyomyositis: increasing recognition in temperate climates. Am J Med 1984;77:768-72.

7 Brown JD, Wheeler B. Pyomyositis: report of 18 cases in Hawaii. Arch Intern Med 1984;44:1749-51.

8 Kallen P, Nies K, Louie J, Keller M, Worthen N, Bayer AS. Tropical pyomyositis. Arthritis Rheum 1982;25:107-10.

9 Smith IM. Vickers AB. Natural history of 338 treated and untreated patients with staphylococcal septicaemia (1936-1955). Lancet 1960;i:1318-22.

10 Cluff L, Reynolds R, Page B, Breckenridge JL. Staphylococcal bacteraemia and altered host resistance. Ann Intern Med 1968;69:859-73

11 Libman H, Arbeit R. Complications associated with Staphylococcus aureus bacteraemia. Arch Intern Med 1984; 144:541-5.

12 Levin MJ, Gardner P, Waldvogel FA. Tropical pyomyositis: an unusual infection due to Staph aureus. $N$ Engl $J$ Med 1971;284:196-8.

13 Schleck WF, Moulton P, Kaiser AB. Pyomyositis: tropical dis ease in a temperate climate. Am J Med 1981;71:900-2.

14 Mitsuyasu R, Gale RP. Bacterial pyomyositis in a patient wit居 aplastic anaemia. Postgrad Med J 1980;56:61-2.

15 Jordan GW, Bauer R, Wong GA, Barry LA. Staphylococcal myositis in a compromised host. Successful treatment with a synergistic combination of nafcilin and gentamicin. West $J$ Med 1976;124:140-3.

16 Alavi IA, Smith EC, Lattoo H. Pyomyositis-tropical and nontropical. N Engl J Med 1971;284:854.

17 Barrett AM, Gresham GA. Acute streptococcal myositis. Lancet 1958;i:347-51.

18 Rogers DW. A case of pyomyositis occurring in London. $\mathrm{Br}$ Med $J$ 1973;3:679.

19 Williams RHP, Thomas P. An unusual case of spontaneous bacterial myositis. Postgrad Med J 1975;51:255-7.

20 Miyake H. Beitrage zur Kenntnis der sogenannten Myositis Infectiosa. Mitteilungen aus den Grenzgebieten der Medizin und Chirurgie 1904;13:155-98.

21 Ashken MH, Cotton RE. Tropical skeletal muscle abscesses (pyomyositis tropicans). Br J Surg 1963;50:846-52.

22 O'Brien DD. Pyomyositis in London. Br Med J 1974;i:78.

23 Taylor JF, Fluck D, Fluck D. Tropical myositis: ultrastructural studies. J Clin Pathol 1976;29:1081-4.

24 Taylor JF, Templeton AC, Henderson B. Pyomyositis: a clinical pathological study based on 19 autopsy cases, Mulago hospital, 1964-1968. East Afr Med J 1970;47:494-501.

25 Fox IH. Hypouricaemic agents in the treatment of gout. Clin Rheum Dis 1977;3:145-58.

26 Goldberg JS, London WL, Nagel DM. Tropical pyomyositis: a case report and review. Pediatrics 1979;63:298-300.

27 Finnerty RU, Vordermark JS, Modarelli RO, Buck AS. Primary psoas abscess: case report and review of literature. $J$ Urol 1981;126:108-9.

28 Peller JS, Bennett RM. Bacterial pyomyositis in a patient with preleukaemia. $J$ Rheumatol 1985;12:185-6.

Request for reprints to: Dr JG Cruickshank, Consultant Microbiologist, Deputy Director, Public Health Laboratory, Church Lane, Heavitree, Exeter EX2 5AD, England. . . , . 。 